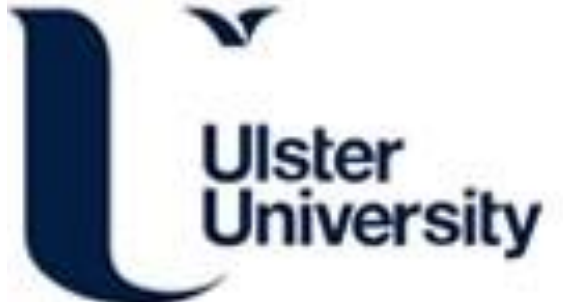

\section{Discriminating at-risk post-MI patients by fractal dimension analysis of the late potential attractor}

Mitchell, RH., \& Escalona, OJ. (1988). Discriminating at-risk post-MI patients by fractal dimension analysis of the late potential attractor. In Unknown Host Publication (Vol. 20, pp. 1573-1575). IEEE.

https://doi.org/10.1109/IEMBS.1998.747193

Link to publication record in Ulster University Research Portal

\section{Published in:}

Unknown Host Publication

Publication Status:

Published (in print/issue): 15/11/1988

DOI:

10.1109/IEMBS.1998.747193

\section{Document Version}

Publisher's PDF, also known as Version of record

\section{General rights}

Copyright for the publications made accessible via Ulster University's Research Portal is retained by the author(s) and / or other copyright owners and it is a condition of accessing these publications that users recognise and abide by the legal requirements associated with these rights.

\section{Take down policy}

The Research Portal is Ulster University's institutional repository that provides access to Ulster's research outputs. Every effort has been made to ensure that content in the Research Portal does not infringe any person's rights, or applicable UK laws. If you discover content in the Research Portal that you believe breaches copyright or violates any law, please contact pure-support@ulster.ac.uk. 


\author{
DISCRIMINATING AT-RISK POST-MI PATIENTS BY FRACTAL \\ DIMENSION ANALYSIS OF THE LATE POTENTIAL ATTRACTOR \\ R.H. Mitchell \& O. Escalona \\ *University of Ulster at Jordanstown, \\ Northern Ireland. \\ e-mail: rh.mitchell@ulst.ac.uk \\ Universidad Simon Bolivar, \\ Caracas, Venuzuela.
}

\begin{abstract}
A novel and reliable approach which quantifies the degree of complexity of Late Potential(LP) activity in the time domain is presented. By defining the LP Attractor in the microvoltage, 3-dimensional space, and then computing the Fractal Dimension $(\delta)$ of the Attractor's trajectory, the degree of complexity of LP can be quantified with a single parameter. $\delta$ may indicate the chaotic behaviour of the terminal activity of the ventricular depolarisation process.

The Fractal Dimension of the LP Attractor in post-MI subjects that are at risk is significantly higher than in postMI subjects with low LP activity $(\mathrm{p}<0.001)$. $\delta$ may be considered as the criterion for discrimination.

Fractal Dimension analysis on LP is a novel diagnostic approach aimed at quantifying their complexity in the microvoltage 3-D space, and may be interpreted as a measure of their chaotic behaviour triggering a catastrophic arrhythmic episode.

Keywords: Chaos; Late Potentials; Fractal Index

\section{INTRODUCTION}

Ventricular late potentials (LP) are delayed depolarisation electrograms of high frequency which may appear in the ST segment or at the terminal low amplitude $(<40 \mu \mathrm{V})$ portion of the QRS complex [1]. Their detection is only possible using a high-resolution ECG recording. Signal averaging of consecutive beats, usually of sinus origin, is used to attenuate the stochastic signal components in the raw ECG, leaving most of the deterministic components for subsequent analysis. LP analysis has become a widely accepted clinical tool for non-invasive detection of patients at risk after myocardial infarction(MI). Significant correclation exists between the presence of LP on the signal-averaged ECG(SAECG) and inducible monomorphic ventricular tachycardia(VT) at programmed electrical stimulation in survivors of MI. Time and frequency domain analysis of LP activity have met with varying degrees of success.

A novel approach emerges by viewing LP activity in the 3-dimensional(3D) microvoltage space in a similar manner to vectorcardiography. It is evident that the behaviour of the 3D trajectory is not a simple
\end{abstract}

geometric object, but one which may be related to fractals[2]. Following this approach, it is possible to consider the terminal dynamic of the ventricular activation process as an instance of deterministic chaos. This interpretation may offer a better way of characterising the dynamics of LP and it is important because the identification of a phenomenon as chaotic may make new therapeutic strategies possible.

In this paper the dynamic behaviour of ventricular late potentials in the signal-averaged, high-pass filtered ECG as recorded using the bipolar orthogonal lead system, $\mathrm{XYZ}$, is characterised by viewing them in the microvoltage 3D space. By comparing the fractal dimension of the LP attractor(the 3D LP geometric curve), its strangeness is quantified with a single parameter, $\delta$. A preliminary study which investigated the relationship between the parameter $\delta$ and inducibility to VT in post-MI patients was conducted and is presented here.

\section{THEORY}

Attractors may be classified using the concept of dimension. The simplest type of dimension is capacity, also referred to as fractal dimension. The concept of fractal dimension was discussed by Mandlebrot[3] centring on the Hausdorff-Besicovitch dimension and by contrasting it to the Euclidean and topological dimension.

The Hausdorff-Besicovitch dimension $\left(\delta_{\mathrm{H}}\right)$ is a real number which can be used to characterise the geometrical complexity of a set of points embedded in a metric space, by measuring its space filling property[3].

The concept of distance between points in space is central to the definition of the Hausdorff-Besicovitch dimension, and therefore of the fractal dimension $\delta$.

In order to give a measure of the size of a set of points, $A$, in space, a test function $h(\varepsilon)=g(d) \varepsilon^{d}$ having side of diameter $\varepsilon$ and dimension $\mathrm{d}$, may be chosen as a "measuring element" and have covered the set to form the measure $\mathbf{M}_{d}=\Sigma h(\varepsilon)$. In general it is found that as $\varepsilon \rightarrow 0$, the measure $\mathbf{M}_{\mathrm{d}}$ is either zero or infinity depending on the choice of $d$. 
The Hausdorff-Besicovitch dimension of A would be the critical dimension for which the measure $\mathbf{M}_{\mathrm{d}}$ changes from zero to infinity:

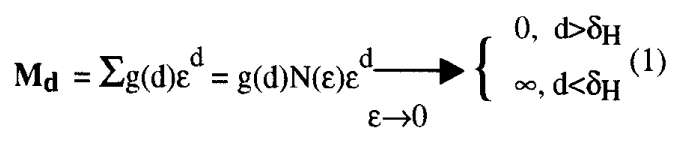

where $N(\varepsilon)$ is the number of measured elements needed to cover the set. The value of $\mathbf{M}_{\mathbf{d}}$ for $\mathrm{d}=\delta_{\mathrm{H}}$ is often finite, but in theory it also may be zero or infinity. It is the position of the jump in of $\mathbf{M}_{\mathbf{d}}$ as a function of $\mathbf{d}$ that is important.

$$
N(\varepsilon) \propto \frac{1}{\varepsilon^{\delta_{H}}}
$$

$\mathbf{M}_{\mathbf{d}}$ is a finite value and therefore Eq.(2) can be rewritten as:

$$
N(\varepsilon) \approx \frac{\beta}{\varepsilon^{\delta}}
$$

where $\beta$ must be a constant value. Therefore;

$$
\log [N(\varepsilon)] \approx-\delta \log (\varepsilon)+\log (\beta)
$$

The fractal dimension $\delta$ may be estimated by finding the absolute value of the slope of the straight line fit of the points of the log-log plot.

\section{METHODS}

A brief description of the data collection and analysis follows.

\section{Signal Recording and Averaging}

Bipolar orthogonal lead ECG recordings ( $\mathrm{X}, \mathrm{Y}$ and $\mathrm{Z}$ ) were digitised at a sampling rate of $2 \mathrm{kHz}$ and 16 -bit resolution. QRS complexes of sinus rhythm were averaged until noise was below $0.3 \mu \mathrm{V}$, or the number of averaged beats exceeded 600 .

\section{Signal Filtering}

High-pass filtering was performed using a $40 \mathrm{~Hz}$ bidirectional $4^{\text {th }}$ order Butterworth digital filter.

\section{Isolation of the LP Attractor}

LP onset and offset points were defined. The end of ventricular activity in each filtered lead was defined as the mid-point of a $5 \mathrm{~ms}$ segment shifted along the ST segment towards the QRS complex in Ims steps until the mean voltage exceed the mean noise level plus 3 times the standard deviation of the noise sample [4].
The start time of the LP attractor was defined on the vector magnitude combination of the three filtered orthogonal leads as the end point of a $5 \mathrm{~ms}$ segment shifted from the end point into the QRS complex in $1 \mathrm{~ms}$ steps until its mean voltage exceeded $40 \mu \mathrm{V}$, or had a peak value above $48 \mu \mathrm{V}$ i.e. $40 \mu \mathrm{V}+20 \%$.

\section{Estimation of $\delta$}

The method for estimating the fractal dimension $\delta$ of $A_{\mathbf{L P}}$ was based on Eq.(4), which assumes the condition that the fractal object is statistically self-similar. In order to find the slope two points need to be known. In the method adopted two wide apart values of $\varepsilon$ can be the following;

$$
\begin{aligned}
& \text { 1. } \varepsilon_{1}=1 \\
& \text { 2. } \varepsilon_{\mathrm{DD}}=\text { the diameter (spheric content) of the } \\
& \text { fractal object. }
\end{aligned}
$$

\section{CLINICAL STUDY}

The purpose of this study was to investigate the relationship between the parameter $\delta$, as a measure of the strangeness of $A_{\mathbf{L P}}$, and inducibility to VT in post-MI patients, thus clarifying the clinical significance of a chaotic-like behaviour.

\section{Patients}

SAECG data was gathered from a control group of 30 healthy volunteers (GROUP 1) and from 30 patients that had myocardial infarction and were VT inducibles at programmed electrical stimulation (GROUP 2).

\section{Analysis}

The fractal dimension $\delta$ of $A_{\mathbf{L P}}$ was computed for each subject in the two Groups according to the methods described above. Significance analysis on the values of $\delta$ between the two clinical Groups was performed using the Student's t-test for independent samples. A value of $\mathrm{p}<$ 0.001 in the two-tailed test level of significance was chosen as the criteria for rejecting the null hypothesis.

Figs. 1 and 2 illustrate examples of the perspective view of $A_{L P}$ in a healthy volunteer from GROUP 1 (Fig.1), and $A_{L P}$ in a post-MI patient (VT inducible) from GROUP 2 (Fig.2).

\section{Results}

Values of $\delta$ in each patient of GROUPS 1 and 2 were computed and the mean and standard deviation of $\delta$ in each GROUP were calculated. The mean value of $\delta$ in healthy volunteers (GROUP 1) was $1.187 \pm 0.0595$. In GROUP 2 the mean value of $\delta$ was $1.321 \pm 0.813$. 
The results of the t-test statistics indicated that parameter $\delta$ was significantly greater in post-MI patients at risk (GROUP 2) than in healthy subjects (GROUP 1) with $p<$ 0.001 .

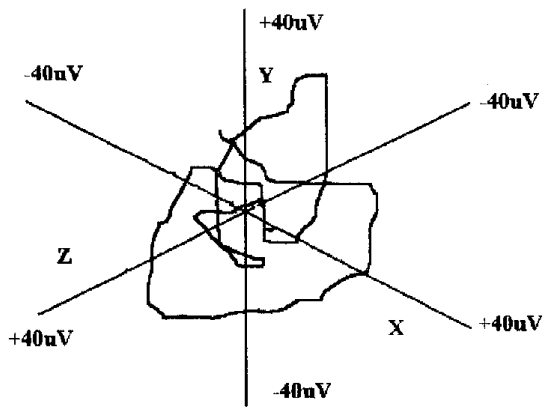

Figure 1. Typical Trace of $\mathbf{A}_{\mathbf{L P}}$ in 3D Voltage Space

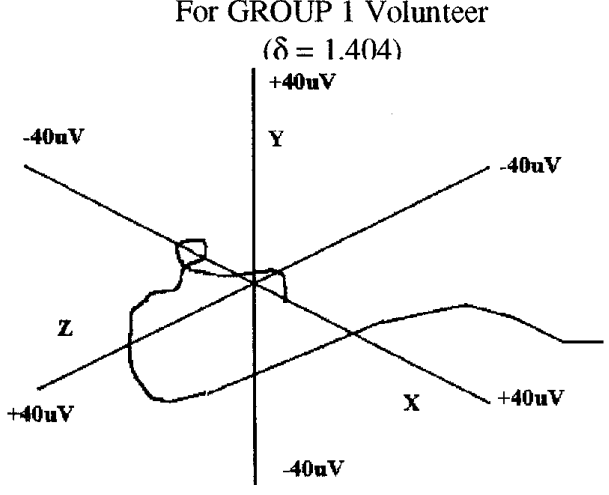

Figure 2. Typical Trace of $\mathbf{A}_{\mathbf{L P}}$ in 3D Voltage Space For GROUP 2 Patient $(\delta=1.163)$

\section{DISCUSSION AND CONCLUSIONS}

In this paper a method of extracting phase-space information from experimental data of late potentials by estimating the fractal dimension of the sampled data has been proposed. In modelling the dynames which may simulate the behaviour of $A_{\mathbf{L P}}$ the values of fractal dimension obtained in the 60 clinical cases may be a useful reference for deciding the the number of differntial equations that would be required in the mathematical model.

Strangeness of the ALP attractor in the infarcted heart may be related to 'turbulence' in the tail portion ' of ventricular activation, produced by anatomicelectrophysiologic obstacles in the pathway of the activation wavefront, inferring the presence of myocardial zones with slow and inhomogeneous activation patterns which may provide the substrate for re-entrant excitation.
Thus, from a cardiopathological viewpoint a 'chaotic-like' behaviour of the isolated LP may result from a disordered distribution of the ventricular depolarisation process, which can be correlated with the subject's susceptibility to ventricular tachycardia.

Values of $\delta$ obtained in the clinical evaluation ranged between 1 and 1.5. From the definition of the HausdorffBesicovitch dimension, in this case, $\delta$ can not be greater than 3. Although the clinical evaluation was carried out with a relatively small population sample, its size was sufficient for the assessment of the clinical significance of $\delta$ as a discriminator of post-MI patients that are at risk. From the results it might be possible to deduce an estimated threshold value for $\delta$ above which a post-MI patient can be classified as susceptible to VT or at-risk. By taking the mean value of $\delta$ in the control group of volunteers and adding twice the standard deviation, a value of about 1.3 is obtained.

\section{REFERENCES}

[1] Balderson, D.E., Harron, D.W.G., Escalona, O., and Mitchell, R.H., "The detection, significance and effect of drugs upon ventricular late potentials," Automedica, Vol. 13, 1991, pp. 67-96.

[2] Escalona, O.J., "A fractal approach to the problem of ventricular late potential detection," Proc. 15 th Ann. Conf. Of the IEEE Eng. Med. \& Biol. Soc., IEEE Press, USA, Vol. 2, 1993, pp. 715-716.

[3] Mandlebrot, B.B.,'The fractal geometry of nature," NY, Freeman, 1982.

[4] Breithardt, G. (Chair), Cain, M.E., El-Sharif, N., Flowers, N., Hombach, V., Janse, M., Simsom, M.B. and Steinbeck, G., "Standards for the analysis of ventricular late potentials using high resolution or signal-averaged electrcardiography: a statement by a Task Force Committee between the European Society of Cardiology, the American Heart Association and the American College of Cardiology," Eur. Heart J., Vol. 12, 1991, pp. 473-480. 